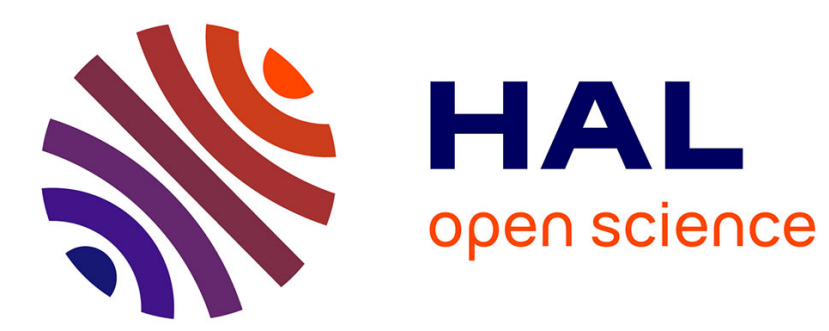

\title{
The generality-specificity of creativity: Exploring the structure of creative potential with EPoC
}

Baptiste Barbot, Maud Besançon, Todd Lubart

\section{To cite this version:}

Baptiste Barbot, Maud Besançon, Todd Lubart. The generality-specificity of creativity: Exploring the structure of creative potential with EPoC. Learning and Individual Differences, 2016, 10.1016/j.lindif.2016.06.005 . hal-01392437

\section{HAL Id: hal-01392437 \\ https://hal.parisnanterre.fr/hal-01392437}

Submitted on 9 Nov 2016

HAL is a multi-disciplinary open access archive for the deposit and dissemination of scientific research documents, whether they are published or not. The documents may come from teaching and research institutions in France or abroad, or from public or private research centers.
L'archive ouverte pluridisciplinaire HAL, est destinée au dépôt et à la diffusion de documents scientifiques de niveau recherche, publiés ou non, émanant des établissements d'enseignement et de recherche français ou étrangers, des laboratoires publics ou privés. 
STRUCTURE OF CREATIVE POTENTIAL

The Generality-Specificity of Creativity: Exploring the structure of creative potential with EPoC.

\title{
Baptiste Barbot, Maud Besançon, Todd Lubart
}

\begin{abstract}
It is increasingly acknowledged that creative potentialinvolvespartly a generalized ability, partly a set of domain-specific abilities, and partly a set of task-specific abilities. We extend and illustrate this viewinastudy of 482 children and adolescents, exploring the extent to which the scores variance ofthe Evaluation of Potential Creativity (EPoC)'seight subtests can be decomposedby five variance components: thinking-process general, thinking-process specific, domain-specific, task-specific, and measurement error. Astructural equation model derived from an extension of the multi-trait multi-method matrix analysisrevealed that (1) the contribution of each variance component depends greatly on the task under consideration, and that (2)the contribution of a general creative thinking-process factor isoverall limited. This study outlines the multidimensional and hierarchical structure of creative potential and the need to measure it with comprehensive test batteries sampling a range of creative tasks, domains and creative thinking-modes.
\end{abstract}

Keywords: EPoC, Creative Potential, Domain-Generality, Domain-specificity, variance partitioning, MTMM. 


\section{STRUCTURE OF CREATIVE POTENTIAL}

\section{The Generality-Specificity of Creativity: Exploring the structure of creative potential with EPoC.}

\section{Introduction}

Creativity is often defined as the ability to produce original work that fits within particular task or domain constraints (e.g., Runco \& Jaeger, 2012; Stein, 1953; Sternberg \& Lubart, 1995).Producing original work relies on creative thinking - the mental operations that lead to new ideas or products regardless of domains of knowledge- which is conceptually a general ability, asare other higher-order mental operations such as intelligence. However, producing an original work that fits the domain or task constraints also requires domain-specific knowledge and skills (unrelated to creative thinking) to make it a valuable contribution.

As a result, there is much debate on the nature of creativity as a "general" as opposed to"domain-specific" ability. This debate stems also from the observation that, though creative thinking may be domain general (e.g., Milgram \& Livne, 2005), onlya few eminent creative individuals have been recognized for their high levels of creativity in multiple subdomains, and eminent creativity in more than one domain remains particularly rare(Baer, 1998; Gray, 1966).Consistently, creativityresearchusing general population samples and samples of individualsengaging in creative activities at a professional level usuallysupport the domainspecificity of creativity. This is evidenced by creative self-beliefsstudies (e.g., creative selfefficacy, or self-perceptions of one's creativity in multiple domains), suggesting often the multidimensional structureof creative self-concepts, organized by content-domains(Karwowski \& Barbot, 2016; Kaufman \& Baer, 2004; Kaufman, Cole, \& Baer, 2009; Kaufman, 2012; Vispoel, 1993), and by studies exploring actual creative behaviors or achievements,also 


\section{STRUCTURE OF CREATIVE POTENTIAL}

outliningthe domain-specificrather than domain-general nature of creativity (e.g., Carson, Peterson, \& Higgins, 2005; Dollinger, 2006; Silvia, Kaufman, \& Pretz, 2009).

Conciliating debates on the general versus domain-specific nature of creativity, hybrid models and multivariate approaches have suggested that creativity resultspartly from a generalized ability, partly from a set of domain-specific abilities, and partly from a set of task-

specific abilities (e.g., Lubart \& Guignard, 2004; Plucker \& Beghetto, 2004).For example, the Amusement Park Theoretical (APT) model of creativity (Baer \& Kaufman, 2005)proposes domain-general initial requirements for creativity and domain-specific outcomes.It is indeed increasingly acknowledged that the ability to produce creative work is partly domain-specific because the nature of the creative work varies with the field (e.g., Barbot, Besançon, \& Lubart, 2011), and even variesas a function of the task within a domain or the constraints within the task (Barbot, Lubart \& Besançon, 2016; Treinen \& Barbot, 2008). A follow-up question is therefore, whether the person-level resources that lead to creative performance in each domain are different or the same across domains (e.g., Kaufman, Plucker, \& Baer, 2008).

In relatedwork (Barbot, Besançon \& Lubart, 2015; Barbot \& Tinio, 2015; Barbot et al., 2011, 2016; Lubart, Besançon, \& Barbot, 2011; Lubart, Zenasni, \& Barbot, 2013), we have posited that there is a similar set of skills and traits that lead to creative performance in each domain, but their optimal combination may vary according to the creative domain andtask under consideration. Specifically, demands of the task interact with aperson's unique combination of resources coming into play in creative work. As a result, individual differences in creative outputs depend on the quality of "fit" between task demands and the person's unique profile of resources.Accordingly, creative potential can be defined as a latent ability, resulting from the confluence of several distinct, but interrelated psychological resources (e.g., Lubart, 1999; 


\section{STRUCTURE OF CREATIVE POTENTIAL}

Sternberg \& Lubart, 1995) that are partly genetically grounded (Barbot, Tan, \& Grigorenko, 2013). These resourcesinclude specific aspects of intelligence, knowledge, cognitive styles, personality, motivation, affect, and physical and socio-cultural contexts (e.g., Lubart et al., 2013). The particular combination of these resources results in an individual's profile of creative potential, which may lead to various degrees of creative outcomes due to hypothetical mechanisms of compensation, thresholds (minimum level of resources needed), interaction between resources (e.g.,Sternberg \& Lubart, 1995), andultimately,interaction between individual resources, creative task demands, time and place (Barbot \& Tinio, 2015). Hence, each individual can be described as having more or less creative potential in a specific domain of work or task (Lubart et al., 2013), and therefore, individualshave multiple potentials for creativitydepending on the fit between their profile of resourcesand various creativetask demands (some of which are domain general, others are domain-specific, and others are uniquely relevant to a particular creative task).

This general framework can help understand the rarity of exceptional levels of creativity in multiple domains because it is not likely that a person'sprofile of resourcesoptimally fits multiple domains or tasks constraints.Instead, most people will show a profile of resources that rarely fit optimally to the demands of a specific creative task, resulting in a vast majority of outputs of "average" creativity (Barbot \& Tinio, 2015).Additionally,a person might never achieve her potential if she doesn't have the opportunity to do so. This view of creative potential urges for the use of assessmentsaccounting for the multifaceted nature of creative potential (multidimensionality of an individual's profile of resources relevant to a given creative task) in multiple domains, in order to address adequately the empirical study of the issue of generalityspecificity of creativity. 


\section{STRUCTURE OF CREATIVE POTENTIAL}

\subsection{Measuring Creative Potential: Integrating Domain-General and Domain-Specific}

\section{Aspects}

As outlined above, the concept of creativity is viewed as a multifaceted and partly a domain-specific ability, which is thought to be trainable.As reviewed in related work (Barbot et al.,2015; Besançon, Lubart, \& Barbot, 2013), several domain-specific training programs have been developed with the objective to enhance creative thinking at elementary and secondary school levels in a number of ways (e.g.,Lynch \& Harris, 2001; Starko, 1995). In order to better target and monitor students' development with these programs, creative potential should be measured by assessment tools tapping into the multidimensionality of the construct (Barbot et al., 2011). Two main paths to the measurement of creative potential have been developed to achieve this endeavor (Lubart et al., 2013): the first is "analytic" (or resource-based) and examines the fit between an individual's resources and creative tasks demand, whereasthe second is "holistic" (or outcome-based) and captures an individual level of creative potential, using task performance in situations simulating various aspects of the creative work. Both approaches shed light on the issue of generality versus domain-specificity of creativity.

\subsubsection{Analytic-Componential approaches}

The "analytic" approach to the evaluation of creative potential combines the assessment of individuals' characteristics and task parameters to identify the specific set of abilities, knowledge, and traits involved in a particular activity, and the relative weights of these different resources needed to yieldhighly creative outcomes (e.g., Caroff \& Lubart, 2012). Therefore, this approach relies greatly on the analysis of a particular task demand. In this vein, expert-elicitation methods have been used to identify the set of resources needed in a particular domain, suchas creative writing (Barbot, Tan, Randi, Santa-Donato, \& Grigorenko, 2012) or managerial 


\section{STRUCTURE OF CREATIVE POTENTIAL}

creativity (Caroff \& Lubart, 2012). Although these approaches have proved useful to identify the most central resources, they may be limited to estimate the relative importance of each resource because various relevant sub-groups of experts tend to value these resourcesdifferently depending on their own experience with the creative outlet under investigation (Barbot, Tan et al., 2012).

Alternatively, we developed a "creative profiler" approach (Lubart et al., 2013) which is a more direct, analytic evaluation of domain-specific potential for creativity. It consists of measuring the likelihood that an individual's multidimensional profile of creative potential fits the optimal profile established for a given creative work. After modelling a "target" profile of relevant resources derived from a group of individuals recognized for their high level of creative outcomes in a given domain, individual profiles are then compared to the target profile using classic statistical measures of distance (e.g., Barbot, Haeffel et al., 2012).

Because this approach is highly domain-, or even task-specific, it may prove efficient for improving thepredictive validity of creative potential measures (within the set of targeted creative outcomes), which represents, thus far, a severe limitation of existing "general" creative potential measures (Barbot et al., 2011; Haensly \& Torrance, 1990; Houtz \& Krug, 1995) often used as predictor of "specific" creative outcomes (e.g., Barbot \& Tinio, 2015; Kaufman et al., 2008; Plucker \& Renzulli, 1999). However, the downside of this approach is thattest scores may not be generalized to creative outcomes other than those already modeled in the targeted profile, unless some specific resources represent the "building-blocks" of any creative activity, which is the position assumed by "holistic" approaches to the evaluation of creative potential.

\subsubsection{Holistic Approaches to the Evaluation of Creative Potential}




\section{STRUCTURE OF CREATIVE POTENTIAL}

Holistic approaches to the evaluation of creative potential involvetasks that simulate various aspects of the creative work. Here, the outcome is not an actual creative accomplishment, but the performance ona standardized task that engage all, or some aspects of a person's creative potential, in one specific domain (e.g., Barbot \& Lubart, 2012) or multiple domains (Lubart et al., 2011; Torrance, 1966). Performance on such tasks may be norm-referenced or criterionreferenced and is sought to elicit conceptually the "building blocks" of the individual's creative potential, traditionally in a unidimensional perspective (i.e., creativity viewed as a unitary construct; e.g., Torrance, 1966) coined "g-factor view" (Barbot \& Tinio, 2015), and more recently, in a multidimensional perspective. Following the later approach, we developed a measure to assess creative potential in children and adolescents: the Evaluation of Potential Creativity (EPoC; Lubart et al., 2011).

EPoC was designed to measure two key creative thinking-process clusters (divergentexploratory and convergent-integrative) in multiple domains (currently verbal-literary and graphic, with forthcoming extensions in social problem-solving, scientific and musical domains). The divergent-exploratory mode of thinking refers to the process of expanding the range of solutions in creative problem solving. Conceptually, this thinking-process cluster involves cognitive components such as flexibility, divergent thinking or selective encoding, and conative components, such as openness to new experiences and intrinsicortask-oriented motivation (e.g., task-oriented motivation highlights the perseverance needed to keep exploring different solutions).The convergent-integrative thinkingprocess cluster refers to theactivity of combining, integrating or synthesizing elements in new ways, another fundamental component of the creative work (Barbot et al., 2011; Lubart et al., 2011).The convergent-integrative cluster conceptually involves cognitive components such as associative thinking (e.g., combining 


\section{STRUCTURE OF CREATIVE POTENTIAL}

remote elements), selective comparison and combination, and conative components such as tolerance for ambiguity (which allows comparisons, metaphors, and the ability to re-interpret a situation), risk-taking, or achievement motivation (needed to complete a product). This cluster is not restricted to Guilford's “convergent thinking” (Guilford, 1950; Guilford, 1967), although it conceptually encompasses some convergent operations needed for the creative work such as "synthesis" and "evaluation of ideas" (see e.g., Cropley, 2006; Lubart, 2001; Osborn, 1953).

Insert Table 1 About Here

To reliably measure both thinking-process clusters in each domain of creative work, a recurrent issue with existing measures (Chen, Himsel, Kasof, Greenberger, \& Dmitrieva, 2006),EPoC consists of two tasks engaging divergent-exploratory thinking processes and two tasks involving convergent-integrative thinking processes, in each domain (further details on each task are provided in the measure section). As a result, EPoC consists of an eight subtest structure yielding four composite indexes representing four "Thinking-Process-Domain unit," namely, "Divergent Graphic” (DG), "Integrative Graphic” (IG), "Divergent Verbal” (DV) and "Integrative Verbal” (IV). This structured framework, represented in Table 1, allowsan individual's profile of creative potential to be derived, showing the relative strengths and weaknesses of the test-taker in each Thinking-Process-Domain unit.Therefore, instead of relying on a unique composite index summing the performance in each subtest (supposing a conceptualization of creative potential as a unitary characteristic), EPoC operationalizes creative potential as simultaneously "domain-specific" and "thinking-process specific," resulting in multiple indicators of creative potential that are thought to be relatively independent. EPoC and 


\section{STRUCTURE OF CREATIVE POTENTIAL}

its structured framework have shown good evidence for construct validity (measurement model fittingreasonablythe normative data collected for the development of norms), and internal consistency of the composite indexes, which were expectedly found to be rather independent (inter-correlations between indexes ranging from .11 to .47 , mean $=.24$; Lubart et al., 2011 ). This relative independence is consistent with prior research showing rather low correlations between creative potential scores in tasks of different natures and/or from different domains (e.g., Baer, 1993; Baer, 1998).

\subsection{Investigating Generality vs. Domain Specificity of Creative Potential: Methodological}

\section{Considerations}

Multiple methodological designs have been used for the study of the generality vs. domain-specificity of creativity, including qualitative analyses of historically eminent creative individuals (Gray, 1966) or transfer designs, in which individuals are trained in a creative domain and then assessed for improvements in another creativity outlet (e.g., Baer, 1996; Barbot et al., 2013). However, the extent to which an individual may achieve similar levels of creative potential across domains and tasks may ultimately depend on the similarity between the tasks used to measure it. Hence, exploring the similarity and differences between multiple creative potential tasksthrough correlational studies design has been an important tradition for the empirical study of the generality-specificity of creative potential.These studies have yielded correlations in the .20 range (seeBaer, 1998's review), often interpreted as an evidence of lack of domain-generality and instead, as evidence for domain-specificity.

Silvia and colleagues(2009)have energetically argued against correlational and factor analytical approaches to the study of domain generality vs. specificity of creativity. The authors have identified several limitations to such approaches. First, linear correlations are sample- 


\section{STRUCTURE OF CREATIVE POTENTIAL}

specific and do not account for population heterogeneity, masking subpopulations that may show different patterns of associations between variables. Second, factor structures tend to favor domain-general models because correlation-based analyses assume that everyone has some levelof creativity, which artificially increases the covariance between variables when individuals tend to have "no creativity" in multiple domains. Third, Silvia and colleaguesindicate that correlational research uses null effects asevidence for domain-specificity, thoughevidence against generality should not be interpreted as evidence in favor of specificity.Indeed, it can also indicate a methodological weaknesses. For exampleChen and colleagues (2006) have pointed out that low correlations among scores from different domains may result from the low reliability of measurements used (yielding attenuated correlations between measures due to measurement error), most of which include only a single creativity task as indicator of a rather broad and multifaceted construct.

Due to these limitations, Silvia and colleagues (2009)have concludedthat latent class analyses and other statistical classification techniques(of individual "profiles") were the best approaches to investigate domaingenerality-specificity. Although this suggestion is consistent with our approach to the evaluation of creative potential as implemented in the Creative Profiler and $\mathrm{EPoC}$ (i.e.,leading to individual profiles of creative potential rather than to an unitary score reflecting overall performance),we argue that both approaches("variable-centered" versus "person-centered" approach)shed a different and complementary light on the issue of domain generality-specificity.The "person-centered" approachuncovers the issue of domain-generalityspecificity at the individual level (one's profile of resources and its interaction with various tasks demands will result in the homogeneity or in the heterogeneity of creativity outcomes in different tasks). Variable-centeredapproaches can help understand the nature of various creative 


\section{STRUCTURE OF CREATIVE POTENTIAL}

tasksdemands (some of which are domain general, others are domain specific, and others are task specific) based on the similarity and differences between tasks (usually interpreted in terms of evidence of domain generality vs. specificity). To this end, powerful extensions of classic factor analyses can help to elicit both general and domain-specific aspects of creativity tasksthrough analyses of common variance. Indeed, test score variance can be partitioned (conceptually) into common, specific, and error variance (Thurstone, 1947), and this principle was further developed in the analysis of the multi-trait multi-method matrix(Campbell \& Fiske, 1959), whichhas led to powerful extensionsinrecent years.

\subsection{Present Study}

In the present study, we are exploring a new analytic perspective for the study of generality-specificity, whichimplements a variance partitioning modelforEPoC (Lubart et al., 2011), a holistic measure of creative potential involving multiple "Convergent-Integrative" and "Divergent-Exploratory" tasks in two content-domains (Verbal-literary and Graphic). Because EPoC's tasks engage multiple Thinking Process-Domain units (i.e., nested nature of creative thinking-processes and domain-specific skills within each task), analyses of common variance and covariance structure between EPoC tasks may be used to isolate the unique contribution of delimited variance components, namely, (1) domain-specific variance (variance uniquely shared by all EPoC tasks in a given content-domain), (2) thinking-process specific variance (variance uniquely shared by all tasks taping into the same underlying thinking-process cluster regardless of content-domain), (3) general creative thinking-process (variance explained by a higher-order thinking-process component underlying the covariance between the first-order thinking-process clusters), and uniqueness (i.e., variancethat is not explained by either domain-specific, thinkingprocess specific cluster, or general thinking-process variance components). Further, uniqueness 


\section{STRUCTURE OF CREATIVE POTENTIAL}

can be decomposed conceptually into (4) measurement error (random source of variance that is not related to anything else) and (5) task-specific variance (remaining variance in a given task that is conceptually related to the unique features of that task).

Although thisapproach is colored by a "hybrid" conception of creative potential, as being simultaneously domain-specific and general, it has the advantage to test simultaneously for the contribution of multiple level of resourcesthat come into play in the creative work, including not only domain-specific and general resources, but also thinking process-specific and task-specific resources, which may explain the relation (or lack thereof) between multiple creative potential tasks. In sum,the present study attempts to combine both the componential-analytic approach (relying on the analysis of "task demands"), and the holistic approach to the evaluation of creative potential, in order to examine the structure of creativity and the generality-specificity issuein a new way.

\section{Method}

\subsection{Participants}

Data analyzed in this study was obtained from a sample of 482 French children and adolescents evaluated as part of the development of the EPoC's French norms (Lubart et al., 2011). Specifically, the sample included $51.5 \%$ girls and $48.5 \%$ boys, with a meanage of 11.7 years old $(\min$ age $=4.1$ years old, $\max$ age 16.1 years old, $\mathrm{SD}=3.8$ years old). All participants were recruited in public schools of Paris and Lyon vicinities.

\subsection{Measure and Procedure}

According to the structured framework presented above (see Table 1), EPoC consists of eight "Convergent-Integrative" and "Divergent-Exploratory" tasks appliedin two contentdomains (Verbal-literary and Graphic). In the Divergent-Exploratory thinking tasks for graphic 


\section{STRUCTURE OF CREATIVE POTENTIAL}

domain (DG index), test-takers must generate as many drawings as possible using a simple abstract shape (DG1 - Abstract Stimulus) or a familiar object (DG2 - Concrete Stimulus) as a starting point, in a limited time (10 minutes). Similarly, divergent-exploratory thinking tasks in the verbal domain (DV index) consist of generating either multiple simple story-endings in response to a unique story-beginning (DV1 - Story Endings), or multiple story-beginnings in answer to a unique story-ending (DV2 - Story Beginnings), in 10 minutes. In contrast, the convergent-integrative tasks in graphic domain (IG index) engage test-takers to produce a complete, original drawing, using at least four out of eight abstract shapes (IG1 - Abstract Stimuli) or familiar objects (IG2 - Concrete Stimuli) provided as a basis for their composition (within the15 minutes allowed for these tasks). Similarly, in the convergent-integrative tasks applied to the verbal-literary domain, test-takers have to produce a complete story either based from a provided story title (IV1 - Story Title), or from the integration of imposed fictional characters (IV2 - Story Characters).

Following EPoC's administration guidelines, the battery was administered in two sessions of 45 minutes, each of which included four tasks (DG1, DV1, IG1, IV1 in the first session, and DG2, DV2, IG2, IV2 in the second session). Both sessions occurred at a two week interval in individual ( $<12$ years old) or group settings $(>12$ years old). Note that all DivergentExploratory thinking tasks are scored for fluency only (number of relevant responses generated in response to the task) and are norm-referenced (performance is compared to a subject's agegroup). The Convergent-Integrative tasks are all criterion-referenced, that is, the creative productions are evaluated with regard to a set of defined rubrics, ranging from "1-low creativity" to "7-high creativity".Per the French norms development procedure, the Consensual Assessment Technique (CAT;Amabile, 1982)was used to score all Convergent-Integrative thinking tasks by 


\section{STRUCTURE OF CREATIVE POTENTIAL}

at least three independent and qualified raters ${ }^{1}$, and $10 \%$ of the Divergent-Exploratory protocols were double-scored by two independent raters to estimate inter-scorer reliability.

\subsection{Data Analyses}

After a series of preliminary analyses examining the distributional features and reliability estimates of scores for each task, distributional features of all variables were homogenized into normal distributions using the Rankit method (e.g.,Solomon \& Sawilowsky, 2009), a highly applicable transformation that can approximately normalize any distribution shape (Bishara \& Hittner, 2012), a particularly desirable feature for planned analyses. Next, we applied a variance partitioning modelfollowed by the Schmid-Leiman Transformation (SLT) to estimate all desired variance components for each EPoC task.

Specifically, the model developed in this study assumed that EPoC scores include both thinking-process specific variance and domain-specific variance, which can be partitioned by analyzing EPoC'sinter-subtest correlation matrix as a Multi-Trait-Multi-Method matrix (MTMM; Campbell \& Fiske, 1959), using a general Confirmatory Factor Analysis (CFA) in a Structural Equation Modeling (SEM) framework. Initially developed to evaluate the construct validity of a set of measures, the MTMM approach was extended in a range of variations, including the correlated-trait-correlated-methods model (Jöreskog, 1971) used in this study to partition variance in $\mathrm{EPoC}$ scores into four components: (1) one reflecting the specific skills involved in a particular content-domain to which a given task belongs ("domain-specific variance" uniquely shared by all tasks in a given content-domain); (2) one related to the underlying thinking-process cluster involved ("thinking process-specific" variance shared by all tasks engagingthe same thinking process cluster irrespective of content-domain); (3) one reflecting a higher-order thinking-process component, underlying both first-order thinking- 


\section{STRUCTURE OF CREATIVE POTENTIAL}

process clusters ("general creative-thinking process"), and (4) uniqueness components (i.e., all uncommon sources of variance). The path diagram of thetested model is depicted in Figure 1.

Insert Figure 1 About Here

As illustrated, each task is logically loaded onto one "domain-specific" factor and one “thinking-process" specific factor. Both thinking-process factors are loaded into the general thinking-process factor, conceptually reflecting a hierarchical structure of creative-thinking processesin linewith "hybrid" approaches to domain generality-specificity, and consistent with the idea of domain-general requirements for creative thinking. Based on the theoretical assumption that the domain-specific set of skills could be oblique (e.g., specific verbal abilities may be involved in graphic-oriented tasks, and conversely, specific graphic or figural abilities may contribute to the performance in verbal task), the correlation between these variance components was freely estimated ${ }^{2}$. However, domain-specific and thinking-process specific factors were assumed to be uncorrelated with one another, so that the variance in each task could be partitioned into two independent components (respective domain-specific factor and respective thinking-process specific factor).

To identify the metric of the model and provide a scale for each latent variable, the factor loading of one observed variable was fixed to 1 (i.e., a marker indicator). In order to prevent identification problems often occurring with MTMM data under the CFA framework (Kenny \& Kashy, 1992), the variance of the higher order thinking-process factor was set to 1 (e.g., Mueller \& Hancock, 2008) and the loadings between the general thinking-process factor and both firstorder factors were set to be equal. Although this approach is practical for the sake of model 


\section{STRUCTURE OF CREATIVE POTENTIAL}

identification, it assumes an equal influence of the general-thinking processesfactor (secondorder) on both thinking-process specific (first-order) factors, andresults in estimates that are not any different than a latent correlation (similar to the one depicted on the domain side). Despite this limitation, modeling the general-thinking processes factor had the advantage of representing the shared variance between both thinking-process specific factors, theoretically representing a common, higher-order thinking-process factor (or, "general requirements" for creativity). Further, the contribution of this higher-order factor in the prediction of observed scores could be estimated.

To do so, we applied a Schmid-Leiman Transformation (SLT) procedure for the decomposition of the second-order factor model (Schmid \& Leiman, 1957), after obtaining model estimates for primary and secondary factor loadings. This procedure initially developed for use within the exploratory factor analysis framework generalizes to CFA in a straightforward manner(Brown, 2006), and is used to calculate the contribution of lower and higher-order factors to the prediction of observed measures. Simply stated, SLT consists of multiplying the completely standardized second-order and correspondingfirst-order loadings to estimate the indirect effect of the second-order factor on each indicator (for more details on this procedure, see e.g., Brown, 2006; Gignac, 2007). In the present study, the SLT was useful to isolate the unique contribution of the "general thinking-process" factor on each task, as well as the variance uniquely explained by the first-order factor (thinking-process specific factor) derived from the residualized primary loadings.

Finally, because the uniqueness of each task contains both measurement error and specific factors tapped by that particular task (Meredith \& Horn, 2001), uniqueness was further partitioned into two components reflecting (4a) measurement errorvariance $\left(1-r_{\mathrm{xx}}\right)$ as well as 


\section{STRUCTURE OF CREATIVE POTENTIAL}

other unmodeled variance interpreted as (4b) "task-specific" variance (subtracting measurement error variancefrom uniqueness variance).

Because EPoC was normed across two developmentally distinct age groups (children and adolescents) the measurement invariance (MI) of the above described model was tested in the Multi-group CFA framework following established MI testing guidelines in the literature, (e.g., Bollen, 1989; Byrne, Shavelson, \& Muthén, 1989). Four typical levels of MIstringencywere tested, each adding a new set of constraints to the previous. The congeneric model was fitted simultaneously to both age group data, with the only constraint of an identical number of factors and Scale-factor loading pattern across groups. Factor loadings were set to be equal across groups in the weak invariant model, and indicators intercepts were also set equal in the strong invariant model. Finally, the strict invariant model added to the previous models the equality of residual variance across age groups.

Granted that data hold the assumption of Missingness at Random (MAR; See preliminary analyses section), Full Information Maximum Likelihood (FIML) algorithm was used to estimate all model parameters using AMOS 22 (Arbuckle, 2013). The fit of this model was assessed based on the most widely used indexes of model fit (e.g., Kline, 2010), namely, the Chi-square tests of fit and its Chi-square to degree of freedom ratio, the normed fit index (NFI), the comparative fit index (CFI), and the root mean square error of approximation (RMSEA) with its $90 \%$ confidence interval.MI decisions were based on the $\Delta \chi^{2}$ and $\Delta$ CFI (value under .01 reflect MI;Byrne, 2010; Cheung \& Rensvold, 2002) using the congeneric model as baseline.

\section{Results}

\subsection{Preliminary Analyses}




\section{STRUCTURE OF CREATIVE POTENTIAL}

Amount of missing data across all variables ranged from $0.6 \%$ (DV1) to $6.4 \%$ (IV1) with an average of $2.9 \%$ across EPoC's tasks. The observed pattern of missing values was completely random as indicated by Little's MCARtest $\left(\chi^{2}[29]=94.01, \mathrm{DF}=77, p=.091\right)^{3}$, allowing for the use of FIML. Next, inter-rater reliability coefficients were computed in order toderive estimates of rater-related measurement error for each EPoC subtest score. Although this is particularly relevant for criterion-referenced subtests such as the convergent-integrative tasks (IV1, IV2, IG1, IG2) that may engage inter-rater differences in the interpretation of scoring rubrics, we also estimated inter-rater reliability for the divergent-exploratory tasks (DV1, DV2, DG1, DG2) that are alsosubject to scoring errors. Cronbach's Alpha was used to estimate inter-rater reliability across all three raters for each convergent-integrative task, and Intra-Class Correlation Coefficients (ICC) were used for inter-rater agreement of both raters who scored the divergentexploratory tasks. As reflected in Table 2, inter-rater reliability was good to excellent with coefficients ranging from .81 (IG1) to .99 (DG1, DG2).

After normalization of the data using the Rankit transformation, preliminary analyses indicated adherence to common statistical guidelines and assumptions for planned analyses. The inter-subtest correlation matrix obtained with FIML (Table 2)was not an identity matrix (Bartlett's test of sphericity 917.9, $\mathrm{df}=28, p<.001$ ), and the Kaiser-Meyer-Olkin statistics $(\mathrm{KMO}=.67$; individualMSAs ranging from .65to .73) in the "middling" range (Kaiser, 1974) suggested that the input data was adequate for factor analysis, although variables were not showing, on average, correlations of high magnitude.

Insert Table 2 About Here 


\subsection{Model Estimation}

Model estimation yielded an excellentfit of the data to the hypothesized modelafter 13iterations $\left(\chi^{2}[16]=23.6, p=.099, \chi 2 / \mathrm{df}=1.47, \mathrm{NFI}=.973, \mathrm{CFI}=.991, \mathrm{RMSEA}[90 \%-\right.$ $\mathrm{CI}]=.031[.000-.057])$ as suggested by common model fit index benchmarks in the literature (e.g.,Kline, 2010).Unstandardized and standardized primary factor loadings of both domainspecific factors and process-specific factors are presented in Table 3.

Insert Table 3 About Here

In addition to these primary factor loadings estimates, the variance of all latent variables in the tested model were estimated to be statistically different from zero $(p<.001$; except for the Graphic factor, $p=.09$ ) and the correlation between both domain-specific factors was estimated to ber $=.24(p=.009)$. Second-order factor loadings of the general thinking process factor into both thinking-process specific factor was $.168(\mathrm{SE}=.042, p<.001)$ resulting in standardized loadings estimate of .403 and .395 for the Convergent-Integrative and the Divergent-Exploratory factors, respectively.

Fit indices of the MI testing for the hypothesized model (Congeneric model: $\chi^{2}$ $[26]=45.4, p=.011, \chi 2 / \mathrm{df}=1.75, \mathrm{CFI}=.977, \mathrm{RMSEA}[90 \%-\mathrm{CI}]=.039[.019-.058] ;$ Weak

invariance model $\chi^{2}[39]=62.4, p=.010, \chi 2 / \mathrm{df}=1.60, \mathrm{CFI}=.972, \mathrm{RMSEA}[90 \%-\mathrm{CI}]=.039$ [.024 - .053]; Strong Invariance model: $\chi^{2}[45]=78.2, p=.002, \chi 2 / \mathrm{df}=1.74, \mathrm{CFI}=.960$, RMSEA [90\%-CI] = .042 [.030 - .055]; Strict Invariance model: $\chi^{2}[58]=108.2, p<.001$, $\chi 2 / \mathrm{df}=1.87, \mathrm{CFI}=.939, \mathrm{RMSEA}[90 \%-\mathrm{CI}]=.044[.032-.056])$ indicated that only the "weak 


\section{STRUCTURE OF CREATIVE POTENTIAL}

invariance" held across age groups $\left(\Delta \chi^{2}{ }_{[d f]}=17.1[13], p=.20, \Delta C F I=.005\right)$, and that the most stringent level of invariance (Strict Invariance) didn't held,but was plausible based on the practical fit indices and the reasonable degradation in fit in comparison with the Congeneric $\operatorname{model}\left(\Delta \chi_{[d f]}^{2}=62.8[32], p=<.001, \Delta C F I=.038\right)$. Because weak invariance is sufficient in many practical applications such as group comparisons of variances and covariances among latent variables (e.g.,Barbot, Hein, Luthar, \& Grigorenko, 2014; Millsap, 2011), subsequent analyses were conducted using the combined sample of children and adolescents ${ }^{4}$.

\subsection{Variance component estimation.}

Estimates of primary and second-orderthinking-process loadings obtained with the overall sample (Table 3) were used as a basis of the Schmidt-Leman Transformation. Specifically, secondary loadings and residualized primary loadings were derived to estimate variance uniquely accounted for by the general thinking-process factor, and both thinkingprocess specific factors. The uniqueness (variance not explained by the thinking process factors or the domain-specific skills factors) was further partitioned into rater-related residual measurement error variance and "task-specific" variance ${ }^{5}$.Figure 2 represents the estimated percentage of variance accounted for by each of the five variance components, for each EPoC subtest.

Insert Figure 2 About Here

As represented in Figure 2, results indicated that the influence of the five variance components depends largely onthe task under consideration. Specifically, variance uniquelyaccounted for by the general creativethinking-processfactor was only $5.5 \%$ on average 


\section{STRUCTURE OF CREATIVE POTENTIAL}

$(\min =1.3 \%, \max =11.7 \%)$ whereasvariance uniquely accounted for by the thinking-process specific factor was $29 \%$ on average $(\min =6.7 \%$, $\max =63.4 \%$; with an average of $34 \%$ for the Divergent-Exploratory component, and $24 \%$ for the Convergent-Integrative component).Variance uniquely accounted for by domain-specific skills ranged from $1 \%$ to $59.1 \%$ (mean $21.1 \%$, with $16 \%$ and $23 \%$ explained variance on average by the domain-specific component in the graphic and verbal domains, respectively), whereas task-specific variance was estimated to average $34.1 \%$ (14.9\% to $50.8 \%$ ). Finally, measurement error accounted for $1 \%$ to $19 \%$ of EPoC scores variance (averaging 10.3\%, plus additional sources of error variance confoundedinto the "task-specific" variance components).

Together, about $35 \%$ of the EPoC scores variance could uniquely be attributed to general and specific creative-thinking processes, whereas $21 \%$ wasuniquely explained by domain-specific variance. The remaining portion of the variance ( $44 \%$ ) was simultaneously explained by both task-specific variance and measurement error. Total variance explained by EPoC underlying factors (domain-specific and thinking-process general and specific factors) ranged from 30\% (IG1: convergent-integrative drawing using abstract stimuli) to 77\% (DG2: divergentexploratory graphic tasks using a concrete stimulus), with an average of $56 \%$. Conversely, variance accounted for by task-specific skills and/or measurement error ranged from $23 \%$ to $70 \%$.

\section{Discussion}

This study explored an extension of a classic MTMM analysis tailored to the study of domain generality-specificity of creativity. Specifically, we explored the structure of creative potential as operationalized and measured with EPoC (Lubart et al., 2011) and provided evidence for the contribution of five sources of influence in creative potential test scores: (1) general 


\section{STRUCTURE OF CREATIVE POTENTIAL}

creative thinking-processes, (2) specific thinking-processes, (3) domain-specific skills, (4) taskspecific skills, and (5) measurement error. This operationalization appeared to adequately fit the "reality of the data" and leads to three main conclusions. First, as there are heterogeneous profiles of individual's creative potential (e.g., Silvia et al., 2009), there are heterogeneous "profiles" of tasks engaging creative potential (in terms of the nature of the resources involved in task performance). Second, the contribution of a general creative-thinking process factor is overall rather limited (at least as it is operationalized and represented in EPoC tasks), explaining no more than $12 \%$ of the variance in each task. Third, evidence for rather large amount of variance accountingfor task-specificity suggests that creative potential tasks demands may require more than general and thinking-process specific abilities, as well as domain-specific skills.

In sum, these results suggest that creative potential tasks may vary on their level of both domain- and task-specific requirements, leaving in some cases limited room to capture creative thinking abilities (i.e., when tasks involve too much domain-specific skills, the general and specific creative thinking processes cannot come into play efficiently). For example, it seems logical that a creative potential task in the field of mechanical engineering may not efficiently elicit general creative thinking process of individuals who have no content-domain knowledge and other specific skills in this field. Even when tasks engage only basic content-domain knowledge and skills (such as in EPoC integrative graphic tasks), domain-specificity may outbalance the contribution of the more general "building -blocks" of creative thinking.

This conclusion has important implications for both interpreting past research and for designing future creativity research and assessment. Indeed, creativity researchers have relied for decades on single indicators of creative potential, essentially, divergent thinking tasks. However, 


\section{STRUCTURE OF CREATIVE POTENTIAL}

our study has suggested that most tasks involving the Divergent-Exploratory clusterare mostly thinking process-specific (rather than thinking-process general), with a large influence of domain-specific abilities especially in the verbal domain. Hence, the use of divergent thinking tasks (especially verbal) may not be sufficient when used as a single indicator of "creative potential," a multidimensional construct in nature. Past research relying on single divergentthinking indicators are not invalidated by this finding, but such research should be re-interpreted in this light (they are not "creativity studies" but studies of divergent-thinking in a particular domain, to not say, in a particular task). For instance, due to the different nature of tasks used, it seems reasonable to observe different developmental patterns as a function of the task used as indicator of the creative potential (Barbot et al., 2016; Besançon \& Lubart, 2008).

Future research on creative potential in general, should attempt to measure it with comprehensive test batteries sampling a range of creative tasks, domains, and thinking processes. This study has demonstrated that EPoC is a promising instrument toward this endeavor. It is consistent with Kaufman and colleagues (2008) call for an "ideal creativity assessment that would be $[\ldots]$ based on a hierarchical model of creativity, $[$ and $]$ posits both domain-general and domain-specific elements" (p. 155).Alternatively,studieson a "general" creative potential relying on a limited set of indicators should use tasks that are less influenced by domain-specific knowledge and skills, so that results from such studies can be meaningfully generalized. This suggests that future research similar to the one presented here is needed to better understand the resources involved in response to aspecific creative potential task's demands. Reciprocally, research focused on domain-specific creative potential should use measures that elicit creative thinking "applied" to the domainof interest, while involving a limited set of domain-specific knowledge or advanced technical skills (e.g., Barbot \& Lubart, 2012). 


\section{STRUCTURE OF CREATIVE POTENTIAL}

\subsection{Study Limitations and Future Directions.}

Although this study has shed a new light on the issue of domain generality-specificity and the structure of creative potential, we must acknowledge several limitations as well as important directions for future research. First, despite several strengths of the statistical approach used in this study, identification difficulties ("only" two domain factors and two thinking-process factors) led to a somewhat over-specified model, with the assumption of an equal contribution of the general creative-thinking factor (higher-order thinking process construct) on both thinkingprocess specific factors (i.e., divergent-exploratory clusters and convergent-integrative clusters). Although the tested model returned an excellentfit to the data for such an assumption, it would be useful to estimate freely the contribution of this higher-order construct on each thinkingprocess specific factor, independently.

To do so, alternative analytical strategies based on the generalizability theory, or Partial Least Square Structural Equation Modeling (PLS-SEM) could prove useful to provide a more accurate estimate of the contribution of a general creative-thinking process factor.However, given the initially low association between divergent and integrative thinking factors (i.e., limited common variance), it is not likely that other analytical strategies would raise the contribution of this general factor and drastically modify the estimates presented here.

More importantly, future studies using the approach proposed here should attempt to include additional indicator variables to improve construct representation of first and secondorder factors, as well as adding indicators representing additional creative thinking processes and domains of creative outlets. This would not only help better estimate the contribution of domaingeneral, domain-specific and task-specific factors in various creative tasks, but would also prevent empirical model identification problems that may arise when estimating parameters of 


\section{STRUCTURE OF CREATIVE POTENTIAL}

models that include few indicators per latent variables and/or multiple loadings per indicator (Kenny \& Kashy, 1992) such as in the variance decomposition model proposed here.Finally, the estimated amount of variance attributed to task-specificity may also have been over-estimated in this study.Indeed, because task-specific variance was estimated by decomposing the uniqueness component into task-specific variance and rater-related measurement error, the task-

specificcomponent may also include sources of measurement-error not accounted for by raterrelated error estimates (e.g., time-samplingerror).

Regardless of the strengths and weakness of the analytical approach presented here, it is essential to situate the results obtained in this study with regard to the sample of tasks and participants available for analyses. That is, the structure of creative potential elicited in this study is "limited" to the creative potential as operationalized with EPoC in its current form, as well as to the sample involved in this study. For example, due to the very nature of divergent vs. integrative tasks in EPoC and their corresponding scoring method (e.g., Integrative tasks are criterion referenced and rely on the consensual assessment of external raters), thinking-process specific variance may be somewhat confounded with method variance.Additionally, the rather weakcorrelation observed between domain-specific components may not be representative of the overlap observed between other domain-specific skills(e.g., musicvs. figural). As stated above, it would be useful to conduct a similar analysis encompassingmore creative potential tasks (including tasks taping into the same creative thinking process and domain, but using different methods), sampling more domains of the creative work, and involving a larger and more diverse study sample. In this view, forthcoming extensions of EPoC in social problem-solving, scientific and musical domains, as well as current norm development in several countries,may proveuseful for the development of a more comprehensive model. 


\section{STRUCTURE OF CREATIVE POTENTIAL}

Among the important directions for extension of this study, much work is needed to empiricallyevaluate the underlying constructs captured by our domain-specific, thinking-process specific, and thinking-process general variancecomponents. Indeed, although the analysis of the MTMM matrix can be logically interpreted in light of the said variance components, it would be useful to examine further the correlates and predictors of each modeled component. For example, although its contribution is clearly limited in the present study, the general creative thinkingprocess factor may capture the very essence of creative thinking, or it could represent other underlying cognitive factors such as intelligence or associative thinking also involved in any creative work. To further examine this assumption, future studies should not only include multiple indicators of creative potential in the same study, but also include external criterion measures to evaluate the divergent validity of each variance components. In this vein, correlates of what we coined "domain-specific factors" should be further explored by introducing several relevant criterion measures of domain-specific skills such as verbal fluency, or perceptual and motor skills.

Finally, in line with Silvia and colleagues (2009) the analytical approach presented in this study could be combined to a person-centered approach in order to explore population heterogeneity and extract latent classes of individuals with structural difference in creative potential. Indeed, there is probably no population with a single "one-size-fits-all" structure of creative potential. Beyond the identification of subgroups of individuals for whom creative potential is mostly general as opposed to subgroups showing a creative potential highly domainspecific, such mixture modeling perspective could be useful in view of exploring structural hypotheses regarding the development of creativity (e.g., extending the multi-group CFA approached here for structural comparisons between age-groups). One such hypothesis isBarbot 


\section{STRUCTURE OF CREATIVE POTENTIAL}

\& Tino (2015)'s, proposing that the level of domain-specificity changes with development through childhood into adulthood (i.e., creative potential may "specialize" in a particular domain or subdomain over time). This “specialization-differentiation” hypothesis (Barbot \& Tinio, 2015; see also Plucker \& Beghetto (2004) could be explicitly tested by a careful evaluation of the longitudinal measurement invariance of the proposed EPoC's Model in a cohort of children followed-up though adulthood, while exploring the percentage of variance accounted for by the general factor at different measurement occasions.

\section{Conclusion}

The issue of generality-specificity of creativity has stimulated the appetites of creativity researchers and methodologists for decades (e.g., Gray, 1966) and although multiple approaches have been used to answerthis question, much research is still needed in this area. The study presented here has applied a variance decomposition method to the eightEPoC's subtests (Lubart et al., 2011). Results have re-emphasized the relevance of correlation-based studies of the generality-specificity controversy, and confirmed that creative potential includes domainspecific, task-specific, thinking-process specific and thinking-process general influences.Acknowledgingthese multiple influences could ultimately help to optimize individuals' creative outcomes in specific areas as a function of their particular "profile" of resources. Hence, this study may pave the way to a new era of generality-specificity research, and important advance for creativity assessment, research, and education.

\section{References}

Amabile, T. M. (1982). Social psychology of creativity: A consensual assessment technique. Journal of Personality and Social Psychology, 43(5), 997-1013.

Arbuckle, J. (2013). Amos 22.0 user's guide. SPSS Incorporated. 


\section{STRUCTURE OF CREATIVE POTENTIAL}

Baer, J. (1993). Creativity and divergent thinking: A task-specific approach. Hillsdale, NJ, England: Lawrence Erlbaum Associates, Inc.

Baer, J. (1996). The effects of task-specific divergent-thinking training. Journal of Creative Behavior, 30, 183-187.

Baer, J. (1998). The case for domain specificity in creativity. Creativity Research Journal, 11, 173-177.

Baer, J., \& Kaufman, J. C. (2005). Bridging generality and specificity: The amusement park theoretical (APT) model of creativity. Roeper Review, 27(3), 158-163.

Barbot, B., Besançon, M., \& Lubart, T. I. (2011). Assessing creativity in the classroom. The Open Education Journal, 4(1), 58-66.

Barbot, B., Besançon, M., \& Lubart T.I. (2015). Creative Potential in Educational Settings: its Nature, Measure, and Nurture. Education 3-13, 43(4), 371-381. Doi:

\section{$10.1080 / 03004279.2015 .1020643$}

Barbot, B., Hein, S., Luthar, S. S., \& Grigorenko, E. L. (2014). Capturing age-group differences and developmental change with the BASC parent rating scales. Journal of Applied Developmental Psychology, 35(4), 294-303. doi: 10.1016/j.appdev.2014.04.003

Barbot, B., Lubart, T. I. \& Besançon, M. (2016). "Peaks, slumps, and bumps”: Individual differences in the development of creativity in children and adolescents. New Directions for Child and Adolescent Development, 151, 27-39. Doi: 10.1002/cad.20152

Barbot, B., Haeffel, G. J., Macomber, D., Hart, L., Chapman, J., \& Grigorenko, E. L. (2012). Development and validation of the delinquency reduction outcome profile (DROP) in a sample of incarcerated juveniles: A multiconstruct/multisituational scoring approach. Psychological Assessment, 24(4), 901-912. doi:10.1037/a0028193 


\section{STRUCTURE OF CREATIVE POTENTIAL}

Barbot, B., \& Lubart, T. I. (2012). Creative thinking in music: Its nature and assessment through musical exploratory behaviors. Psychology of Aesthetics, Creativity, and the Arts, 6(3), 231242. doi:10.1037/a0027307

Barbot, B., Randi, J., Tan, M., Levenson, C., Friedlaender, L., \& Grigorenko, E. L. (2013). From perception to creative writing: A multi-method pilot study of a visual literacy instructional approach. Learning and Individual Differences, 28, 167-176.

doi:10.1016/j.lindif.2012.09.003

Barbot, B., Tan, M., \& Grigorenko, E. L. (2013). The genetics of creativity: The generative and receptive sides of the creativity equation. In O. Vartanian, A. Bristol \& J. C. Kaufman (Eds.), The neuroscience of creativity (pp. 71-93) MIT Press.

Barbot, B., Tan, M., Randi, J., Santa-Donato, G., \& Grigorenko, E. L. (2012). Essential skills for creative writing: Integrating multiple domain-specific perspectives. Thinking Skills and Creativity, 7(3), 209-223. doi:10.1016/j.tsc.2012.04.006

Barbot, B. \& Tinio, P.P.L (2015). Where is the "g" in "creativity"? A specializationdifferentiation hypothesis. Frontiers in Human Neuroscience. 8:1041. doi:10.3389/fnhum.2014.01041

Besançon, M., \& Lubart, T. I. (2008). Differences in the development of creative competencies in children schooled in diverse learning environments. Learning and Individual Differences, 18(4), 381-389.

Besançon, M., Lubart, T. I., \& Barbot, B. (2013). Creative giftedness and educational opportunities. Educational and Child Psychology., 30(2), 79-88. 


\section{STRUCTURE OF CREATIVE POTENTIAL}

Bishara, A. J., \& Hittner, J. B. (2012). Testing the significance of a correlation with nonnormal data: Comparison of pearson, spearman, transformation, and resampling approaches. Psychological Methods, 17(3), 399-417.

Bollen, K. A., \& Bollen, K. A. (1989). Structural equations with latent variables. New York: Wiley.

Brown, T. A. (2006). Confirmatory factor analysis for applied research Guilford Press. Byrne, B. (2010). Structural equation modeling using AMOS (Second ed.). New York: Routledge.

Byrne, B., Shavelson, R., \& Muthén, B. (1989). Testing for the equivalence of factor covariance and mean structures: The issue of partial measurement invariance. Psychological Bulletin, 105(3), 456-466. doi:10.1037/0033-2909.105.3.456

Campbell, D. T., \& Fiske, D. W. (1959). Convergent and discriminant validation by the multitrait-multimethod matrix. Psychological Bulletin, 56(2), 81-105. doi:10.1037/h0046016

Caroff, X., \& Lubart, T. I. (2012). Multidimensional approach to detecting creative potential in managers. Creativity Research Journal, 24(1), 13-20. doi:10.1080/10400419.2012.652927

Carson, S. H., Peterson, J. B., \& Higgins, D. M. (2005). Reliability, validity, and factor structure of the creative achievement questionnaire. Creativity Research Journal, 17, 37-50.

Chen, C., Himsel, A., Kasof, J., Greenberger, E., \& Dmitrieva, J. (2006). Boundless creativity: Evidence for the domain generality of individual differences in creativity. The Journal of Creative Behavior, 40(3), 179-199. doi:10.1002/j.2162-6057.2006.tb01272.x

Cheung, G. W., \& Rensvold, R. B. (2002). Evaluating goodness-of-fit indexes for testing measurement invariance. Structural Equation Modeling: A Multidisciplinary Journal, 9(2), 233-255. 


\section{STRUCTURE OF CREATIVE POTENTIAL}

Cropley, A. (2006). In praise of convergent thinking. Creativity Research Journal, 18(3), 391404. doi:10.1207/s15326934crj1803_13

Dollinger, S. J. (2006). Autophotographic individuality predicts creativity: A Seven-Year Follow-up. The Journal of Creative Behavior, 40(2), 111-124.

Gignac, G. E. (2007). Multi-factor modeling in individual differences research: Some recommendations and suggestions. Personality and Individual Differences, 42(1), 37-48.

Gray, C. E. (1966). A measurement of creativity in western civilization. American Anthropologist, 68(6), 1384-1417.

Guilford, J. P. (1950). Creativity. The American Psychologist, 5, 444-454.

Guilford, J. P. (1967). The nature of human intelligence. New York, NY: McGraw-Hill.

Haensly, P. A., \& Torrance, E. P. (1990). Assessment of creativity in children and adolescents. In C. R. Reynolds, \& R. W. Kamphaus (Eds.), Handbook of psychological and educational assessment of children: Intelligence and achievement (pp. 697-722). New York: Guilford Press.

Houtz, J., \& Krug, D. (1995). Assessment of creativity: Resolving a mid-life crisis. Educational Psychology Review, 7(3), 269-300. doi:10.1007/BF02213374

Jöreskog, K. G. (1971). Statistical analysisof sets of congeneric tests. Psychometrika, 36(2), 109-133. doi: 10.1007/BF02291393

Kaiser, H. (1974). An index of factorial simplicity.Psychometrika, 39(1), 31-36. doi:10.1007/BF02291575 


\section{STRUCTURE OF CREATIVE POTENTIAL}

Karwowski, M., \& Barbot, B. (2016). Creative self-beliefs: Their nature, development, and correlates. In J. C. Kaufman, \& J. Baer (Eds.), Creativity and reason in cognitive development (Second ed., pp. 302-326). Cambridge University Press.

Kaufman, J. C. (2012). Counting the muses: Development of the kaufman domains of creativity scale (K-DOCS). Psychology of Aesthetics, Creativity, and the Arts, 6(4), 298-308. doi:10.1037/a0029751

Kaufman, J. C., \& Baer, J. (2004). Sure, I'm creative--but not in mathematics!: Self-reported creativity in diverse domains. Empirical Studies of the Arts, 22(2), 143-155. doi:10.2190/26HQ-VHE8-GTLN-BJJM

Kaufman, J. C., Cole, J. C., \& Baer, J. (2009). The construct of creativity: Structural model for Self-Reported creativity ratings. The Journal of Creative Behavior, 43(2), 119-134.

Kaufman, J. C., Plucker, J. A., \& Baer, J. (2008). Essentials of creativity assessment. Hoboken, NJ: Wiley.

Kenny, D. A., \& Kashy, D. A. (1992). Analysis of the multitrait-multimethod matrix by confirmatory factor analysis. Psychological Bulletin, 112(1), 165-172. doi:10.1037/00332909.112.1.165

Kline, R. B. (2010). Principles and practice of structural equation modeling (3rd ed.). New York, NY: The Guilford Press.

Lubart, T. I. (1999). Componential models of creativity. In M. A. Runco, \& S. Pritzer (Eds.), (pp. 295-300). New York:NY: Academic Press.

Lubart, T. I. (2001). Models of the creative process: Past, present and future.13(3), 295-308.

Lubart, T. I., Besançon, M., \& Barbot, B. (2011). Evaluation du potentiel créatif (EPoC). Paris: Editions Hogrefe France. 


\section{STRUCTURE OF CREATIVE POTENTIAL}

Lubart, T. I., \& Guignard, J. (2004). The generality-specificity of creativity: A multivariate approach. In R. J. Sternberg, E. L. Grigorenko \& L. Singer (Eds.), Creativity: From potential to realization (pp. 43-56). Washington, DC: American Psychological Association.

Lubart, T. I., Zenasni, F., \& Barbot, B. (2013). Creative potential and its measurement. International Journal of Talent Development and Creativity, 1(2), 41-51.

Lynch, M. D., \& Harris, C. R. (2001). Fostering creativity in children, K-8: Theory and practice. Needham Heights, MA: Allyn \& Bacon.

Meredith, W., \& Horn, J. (2001). The role of factorial invariance in modeling growth and change. In M. Linda, \& A. G. Sayer (Eds.), New methods for the analysis of change. (pp. 203-240) Washington, DC, US: American Psychological Association. doi:10.1037/10409007

Milgram, R., \& Livne, N. (2005). Creativity as a general and a domain-specific ability: The domain of mathematics as an exemplar. In J. C. Kaufman, \& J. Baer (Eds.), Creativity across domains: Faces of the muse (pp. 187-204). Mahwah, NJ London: LEA publishers.

Millsap, R. E. (2011). Statistical approaches to measurement invariance. Routledge, New York.

Mueller, R. O., \& Hancock, G. R. (2008). Best practices in structural equation modeling. In J. Osborne (Ed.), Best practices in quantitative methods (pp. 488-508). Thousand Oaks, CA: Sage.

Osborn, A. F. (1953). Applied imagination. Oxford, England: Scribner's.

Plucker, J. A., \& Beghetto, R. A. (2004). Why creativity is domain general, why it looks domain specific, and why the distinction does not matter. In R. J. Sternberg, E. L. Grigorenko \& J. L. Singer (Eds.), Creativity: From potential to realization (pp. 153-163). Washington DC: American Psychological Association. 


\section{STRUCTURE OF CREATIVE POTENTIAL}

Plucker, J. A., \& Renzulli, J. S. (1999). Psychometric approaches to the study of human creativity. In R. J. Sternberg (Ed.), Handbook of creativity (pp. 35-61). Cambridge, UK: Cambridge University Press.

Runco, M. A., \& Jaeger, G. J. (2012). The standard definition of creativity. Creativity Research Journal, 24(1), 92-96. doi:10.1080/10400419.2012.650092

Schmid, J., \& Leiman, J. M. (1957). The development of hierarchical factor solutions. Psychometrika, 22(1), 53-61. doi:10.1007/BF02289209

Silvia, P. J., Kaufman, J. C., \& Pretz, J. E. (2009). Is creativity domain-specific? latent class models of creative accomplishments and creative self-descriptions. Psychology of Aesthetics, Creativity, and the Arts, 3(3), 139-148. doi:10.1037/a0014940

Solomon, S. R., \& Sawilowsky, S. S. (2009). Impact of rank-based normalizing transformations on the accuracy of test scores. Journal of Modern Applied Statistical Methods, 9(2), 448462.

Starko, A. J. (1995). Creativity in the classroom: Schools of curious delight. New York, NY: Longman.

Stein, M. I. (1953). Creativity and culture. Journal of Psychology: Interdisciplinary and Applied, 36, 311-322.

Sternberg, R. J., \& Lubart, T. I. (1995). Defying the crowd: Cultivating creativity in a culture of conformity. New York, NY: Free Press.

Thurstone, L. L. (1947). Multiple-factor analysis; a development and expansion of the vectors of mind. Chicago: University of Chicago Press. 


\section{STRUCTURE OF CREATIVE POTENTIAL}

Torrance, E. P. (1966). The torrance tests of creative thinking-norms-technical manual research edition-verbal tests, forms $A$ and B-figural tests, forms $A$ and B. Princeton, NJ: Personnel Press.

Treinen, E., \& Barbot, B. (2008). Effect of stimuli in figural divergent thinking abilities among adolescent. Conference of the International Center for Innovation in Education (ICIE), Paris, France.

Vispoel, W. P. (1993). The development and validation of the arts self-perception inventory for adolescents. Educational and Psychological Measurement, 53(4), 1023-1033. doi:10.1177/0013164493053004015 


\section{STRUCTURE OF CREATIVE POTENTIAL}

\section{Footnotes}

Note 1: Graduate-level students were trained to identify the seven defined rubrics on a pilot sample of drawings and stories initially scored by the authors of EPoC and were given feedbacks regarding their severity, discrimination and accuracy until they reached a satisfactory level of agreement.

Note 2:Contrary to the thinking-process specific level that is conceptually supposed to be underlined by a "general creative thinking process factor", we favored domain specific skills correlations to represent the contribution of common skills across the verbal and graphic tasks, rather than a second-order factor that would represent "domain general skill(s) independent from creative thinking" (since the creative thinking process variance is captured on the process side). Such second-order factor would make little conceptual sense, especially if adding indicators of creative potential in domains other than verbal and graphic (what generalized skill that is not related to creative thinking could be common to creative tasks across domains?).

Note 3:Although, the pattern of missingness is considered MCAR allowing for the use of FIML, caution is warranted when interpreting the estimates of the tested model,given that the $p$ value of the Little's MCAR test (.091) is close to the critical alpha level of .05.

Note 4: Given that invariance level was sufficient to generalize reasonably the estimates of interest across both age groups, we have not examined further potential sources of misfit related to the more stringently invariant models, which could be accomplished in future work using a partial invariance testing (Byrne, Shavelson, \& Muthén, 1989). 


\section{STRUCTURE OF CREATIVE POTENTIAL}

Note 5: Hence, note that our task-specific variance estimate may include not only task-specific variance sources, but also measurement-error variance sources not accounted for by rater-related error estimates. 


\section{STRUCTURE OF CREATIVE POTENTIAL}

Table 1.

EPoC Structured Framework for Task Sampling

\section{Thinking Process}

\begin{tabular}{ccc}
\cline { 2 - 3 } Domain & Divergent-Exploratory & Convergent-Integrative \\
\hline Graphic & DG1 - Abstract Stimulus & IG1 - Abstract Stimuli \\
& DG2 - Concrete Stimulus & IG2 - Concrete Stimuli \\
\hline Verbal & DV1 - Story Endings & IV1 - Story Title \\
& DV2 - Story Beginnings & IV2 - Story Characters \\
\hline
\end{tabular}

Notes. DG = Divergent-Graphic; IG = Integrative Graphic; DV = Divergent Verbal; IV = Integrative Verbal. Each "Thinking-Process-Domain Unit" forms an index (e.g., DG index = $\mathrm{DG} 1+\mathrm{DG} 2)$. 
STRUCTURE OF CREATIVE POTENTIAL

Table 2

EPoC Scale Scores Reliability Coefficients and Inter-Subtests Correlation Matrix (FIML estimates).

\begin{tabular}{|c|c|c|c|c|c|c|c|c|c|c|}
\hline & M & $\mathrm{SD}$ & DG1 & DG2 & DV1 & DV2 & IG1 & $\mathrm{IG} 2$ & IV1 & IV2 \\
\hline DG1 - Abstract Stimulus & 5.58 & 3.31 & $(.99)^{\mathrm{a}}$ & & & & & & & \\
\hline DG2 - Concrete Stimulus & 4.67 & 3.12 & $.64 * * *$ & $(.99)^{\mathrm{a}}$ & & & & & & \\
\hline DV1 - Story Endings & 3.82 & 3.77 & $.34 * * *$ & $.36 * * *$ & $(.98)^{\mathrm{a}}$ & & & & & \\
\hline DV2 - Story Beginnings & 2.66 & 2.29 & $.31 * * *$ & $.42 * * *$ & $.63 * * *$ & $(.90)^{\mathrm{a}}$ & & & & \\
\hline IG1 - Abstract Stimuli & 3.66 & 1.34 & $.16^{* * *}$ & $.10^{*}$ & .05 & .09 & $(.81)^{\mathrm{b}}$ & & & \\
\hline IG2 - Concrete Stimuli & 3.53 & 1.25 & $.18 * * *$ & $.15^{* *}$ & $.16^{* * *}$ & $.13 * *$ & $.39 * * *$ & $(.82)^{\mathrm{b}}$ & & \\
\hline IV1 - Story Title & 3.10 & 1.33 & $.17 * * *$ & $.10^{*}$ & $.10^{*}$ & $.11^{*}$ & $.28 * * *$ & $.20 * * *$ & $(.84)^{\mathrm{b}}$ & \\
\hline IV2 - Story Characters & 3.05 & 1.24 & $.14^{* *}$ & .05 & $.11 * *$ & $.17 * * *$ & $.31 * * *$ & $.23 * * *$ & $.46^{* * *}$ & $(.85)^{\mathrm{b}}$ \\
\hline
\end{tabular}

Notes. $N=482$. Coefficients in parenthesis indicate reliability coefficients.; ${ }^{\mathrm{a}}=$ Intra Class Correlation Coefficient $($ ICC $) ;{ }^{\mathrm{b}}=$

Cronbach's Alpha;Correlation matrix shows FIML estimates of the correlations obtained using the normal scores (Rankit

transformation) as input data. The matrix obtained with the non-transformed data was highly similar to the one presented here (Box's

$\mathrm{M}=7.58, p=1) ; * p<.05, * * p<.01, * * * p<.001$ 
Table 3.

Parameter Estimates for Domain-Specific and Thinking-Process Specific Factor Loadings

\begin{tabular}{|c|c|c|c|c|c|c|}
\hline \multirow[b]{3}{*}{ Subtest } & \multirow{2}{*}{\multicolumn{3}{|c|}{$\begin{array}{c}\text { Domain-Specific } \\
\text { Loadings }\end{array}$}} & \multirow{2}{*}{\multicolumn{3}{|c|}{$\begin{array}{l}\text { Thinking-Process } \\
\text { Specific Loadings }\end{array}$}} \\
\hline & & & & & & \\
\hline & Unst. & $S E$ & St. & Unst. & $S E$ & St. \\
\hline DG1 - Abstract Stimulus & .237 & .153 & .180 & $1.625 * *$ & .188 & .703 \\
\hline DG2 - Concrete Stimulus & .192 & .133 & .148 & $1.982 * *$ & .265 & .867 \\
\hline IG1 - Abstract Stimuli & .454 & .269 & .347 & 1 & - & .426 \\
\hline IG2 - Concrete Stimuli & 1 & - & .769 & $.657 * *$ & .136 & .282 \\
\hline DV1 - Story Endings & 1 & - & .665 & $.904^{* *}$ & .095 & .401 \\
\hline DV2 - Story Beginnings & $.994 * *$ & .273 & .673 & 1 & - & .452 \\
\hline IV1 - Story Title & .105 & .090 & .068 & $1.557 * *$ & .261 & .659 \\
\hline IV2 - Story Characters & $.238 *$ & .093 & .156 & $1.578 * *$ & .264 & .674 \\
\hline
\end{tabular}

Notes. $\mathrm{N}=482$. Unst. = unstandardized estimates; $\mathrm{SE}=$ Standard error of the unstandardized estimate; St. $=$ Standardized estimate.Second-order factor loadings of the general thinking process factor $=.168(\mathrm{SE}=.042, p<.001 ;$ Standardized loadings $=.403$ and .395 for the Convergent-integrative and the Divergent-exploratory Thinking- process factors, respectively). 
RUNNING HEAD: STRUCTURE OF CREATIVE POTENTIAL

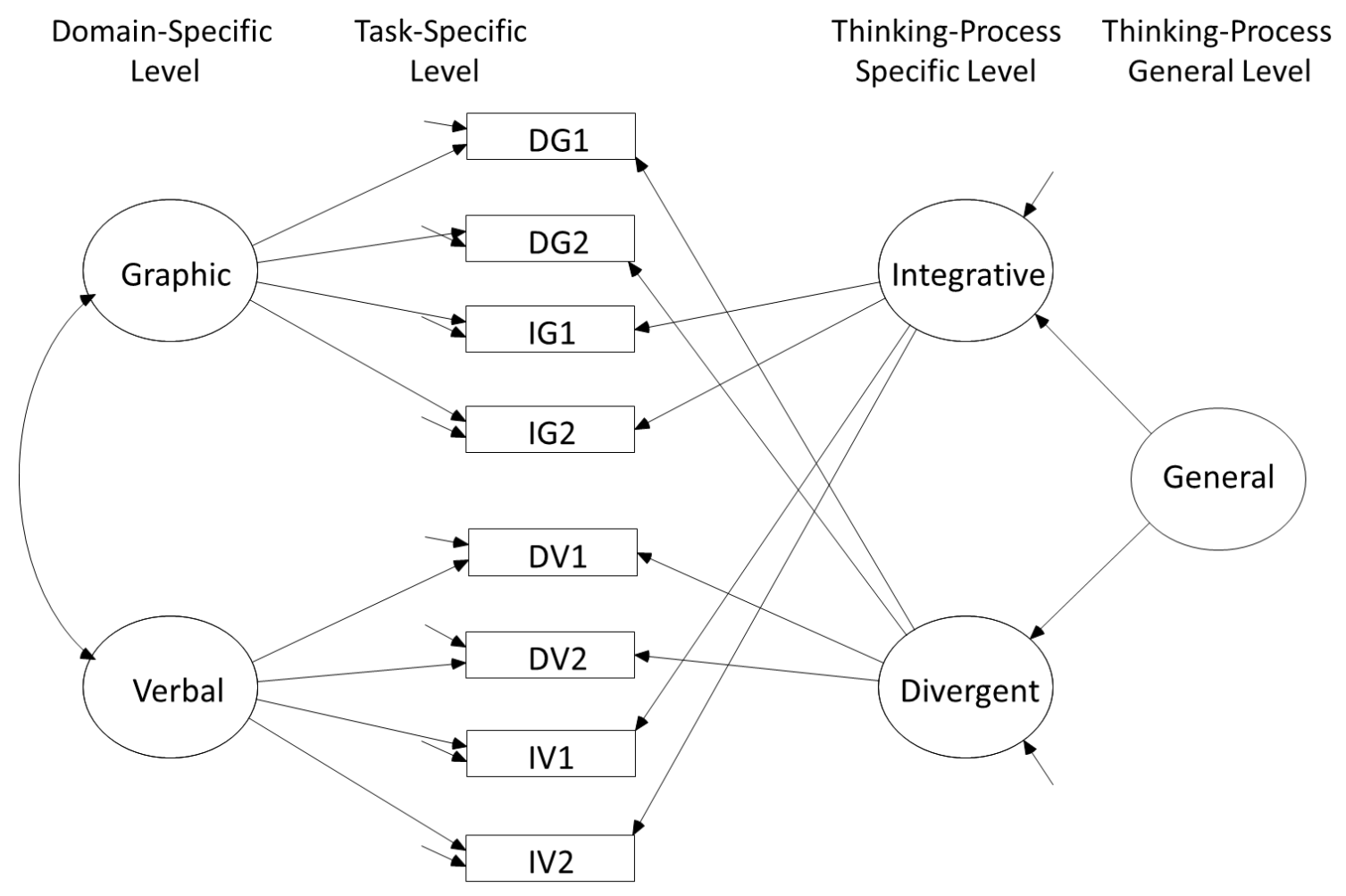

Figure 1.

Multi-Process Multi-Domain Model for Variance Partitioning of EPoC tasks, as specified in the Confirmatory Factor Analysis. 
STRUCTURE OF CREATIVE POTENTIAL

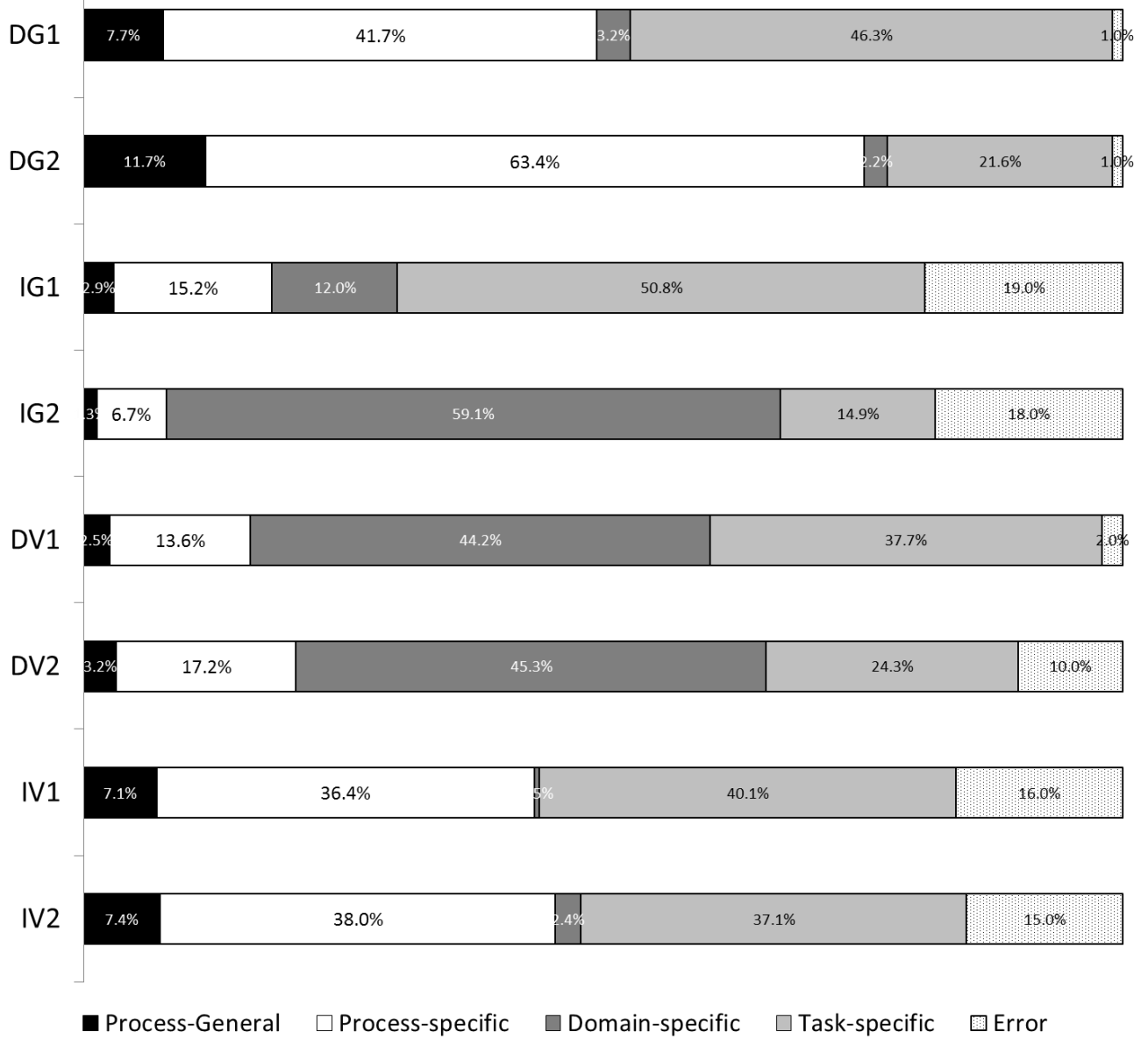

Figure 2.

Estimated Percentage Variance Explained by each Variance Component for each EPoC Task 